\title{
Dealing Crisis Management using AI
}

\author{
Yew Kee Wong \\ School of Information Engineering, HuangHuai University, Henan, China
}

\begin{abstract}
Artificial intelligence has been a buzz word that is impacting every industry in the world. With the rise of such advanced technology, there will be always a question regarding its impact on our social life, environment and economy thus impacting all efforts exerted towards sustainable development. In the information era, enormous amounts of data have become available on hand to decision makers. Big data refers to datasets that are not only big, but also high in variety and velocity, which makes them difficult to handle using traditional tools and techniques. Due to the rapid growth of such data, solutions need to be studied and provided in order to handle and extract value and knowledge from these datasets for different industries and business operations. Numerous use cases have shown that AI can ensure an effective supply of information to citizens, users and customers in times of crisis. This paper aims to analyse some of the different methods and scenario which can be applied to AI and big data, as well as the opportunities provided by the application in various business operations and crisis management domains.
\end{abstract}

\section{KEYWORDS}

Artificial Intelligence, Big Data, Business Operations, Crisis Management

\section{INTRODUCTION}

Artificial intelligence (AI) is a way of making a computer, a computer-controlled robot, or a software think intelligently, in the similar manner the intelligent humans think. AI is accomplished by studying how human brain thinks, and how people learn, decide, and work while trying to solve a problem, and then using the outcomes of this study as a basis of developing intelligent software and systems [1]. AI is a science and innovation based on disciplines such as Computer Science, Biology, Psychology, Linguistics, Mathematics, and Engineering. A major thrust of $\mathrm{AI}$ is in the development of computer functions associated with human intelligence, for example, reasoning, learning, and problem solving. Out of the following areas, one or multiple areas can contribute to build an intelligent system [2]. This paper aims to analyse some of the use of big data for the AI development and its applications in various business operations and crisis management.

\section{What IS Big DATa}

The Big data refers to significant volumes of data that cannot be processed effectively with the traditional applications that are currently used. The processing of big data begins with raw data that isn't aggregated and is most often impossible to store in the memory of a single computer. A buzzword that is used to describe immense volumes of data, unstructured, structured and semistructured, big data can inundate a business on a day-to-day basis. Big data is used to analyse insights, which can lead to better decisions and strategic business moves [3]. The definition of big data: "Big data is high-volume, and high-velocity or high-variety information assets that demand cost-effective, innovative forms of information processing that enable enhanced insight, 
decision making, and process automation." The characteristics of Big Data are commonly referred to as the four Vs:

\section{Volume of Big Data}

The volume of data refers to the size of the data sets that need to be analysed and processed, which are now frequently larger than terabytes and petabytes. The sheer volume of the data requires distinct and different processing technologies than traditional storage and processing capabilities. In other words, this means that the data sets in Big Data are too large to process with a regular laptop or desktop processor. An example of a high-volume data set would be all credit card transactions on a day within Asia.

\section{Velocity of Big Data}

Velocity refers to the speed with which data is generated. High velocity data is generated with such a pace that it requires distinct (distributed) processing techniques. An example of a data that is generated with high velocity would be Instagram messages or Wechat posts.

\section{Variety of Big Data}

Variety makes Big Data really big. Big Data comes from a great variety of sources and generally is one out of three types: structured, semi structured and unstructured data. The variety in data types frequently requires distinct processing capabilities and specialist algorithms. An example of high variety data sets would be the CCTV audio and video files that are generated at various locations in a city.

\section{Veracity of Big Data}

Veracity refers to the quality of the data that is being analysed. High veracity data has many records that are valuable to analyse and that contribute in a meaningful way to the overall results. Low veracity data, on the other hand, contains a high percentage of meaningless data. The nonvaluable in these data sets is referred to as noise. An example of a high veracity data set would be data from a medical experiment or trial.

Data that is high volume, high velocity and high variety must be processed with advanced tools (analytics and algorithms) to reveal meaningful information. Because of these characteristics of the data, the knowledge domain that deals with the storage, processing, and analysis of these data sets has been labelled Big Data [4]. 


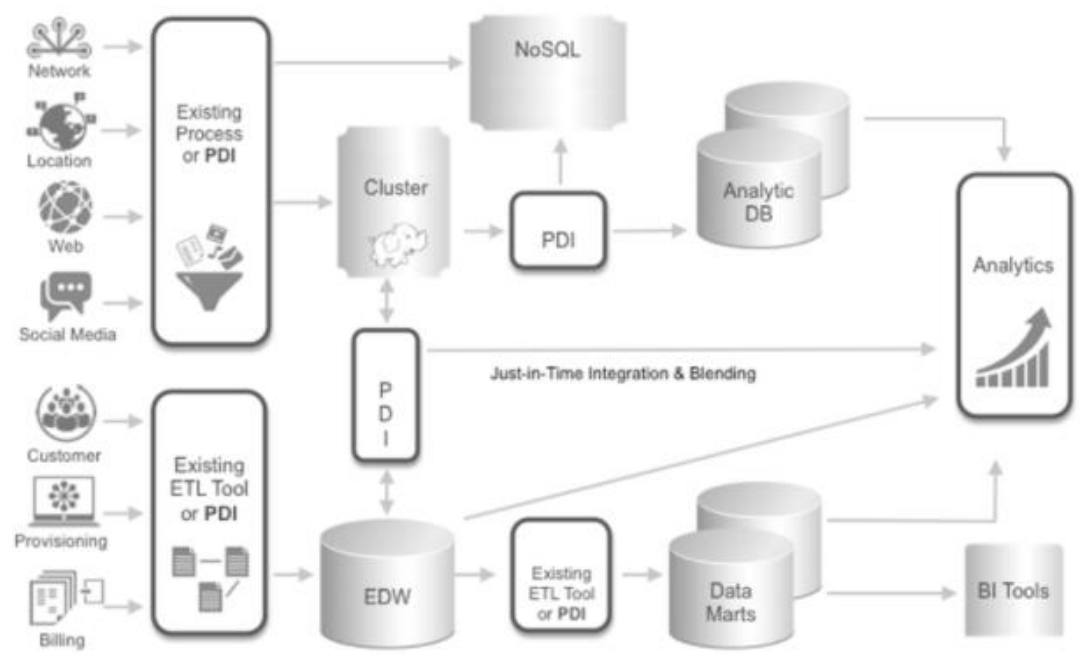

Figure 1. Big Data Architecture. (arccil.com)

\subsection{Types of Big Data}

There are 3 types of big data; unstructured data, structured data and semi-structured data.

\section{Unstructured data:}

Any data with unknown form or the structure is classified as unstructured data.

\section{Structured data:}

Any data that can be stored, accessed and processed in the form of fixed format is termed as a 'structured' data.

\section{Semi-structured data:}

Semi-structured data can contain both the forms of data.

Dealing with unstructured and structured data, data science is a field that comprises everything that is related to data cleansing, preparation, and analysis. Data science is the combination of statistics, mathematics, programming, problem-solving, capturing data in ingenious ways, the ability to look at things differently, and the activity of cleansing, preparing, and aligning data [5]. This umbrella term includes various techniques that are used when extracting insights and information from data.

\section{Big data benefits:}

- Big data makes it possible for you to gain more complete answers because you have more information.

- More complete answers mean more confidence in the data, which means a completely different approach to tackling problems. 


\subsection{What is Big Data Analytics}

Data analytics involves applying an algorithmic or mechanical process to derive insights and running through several data sets to look for meaningful correlations. It is used in several industries, which enables organizations and data analytics companies to make more informed decisions, as well as verify and disprove existing theories or models [6] [7]. The focus of data analytics lies in inference, which is the process of deriving conclusions that are solely based on what the researcher already knows.

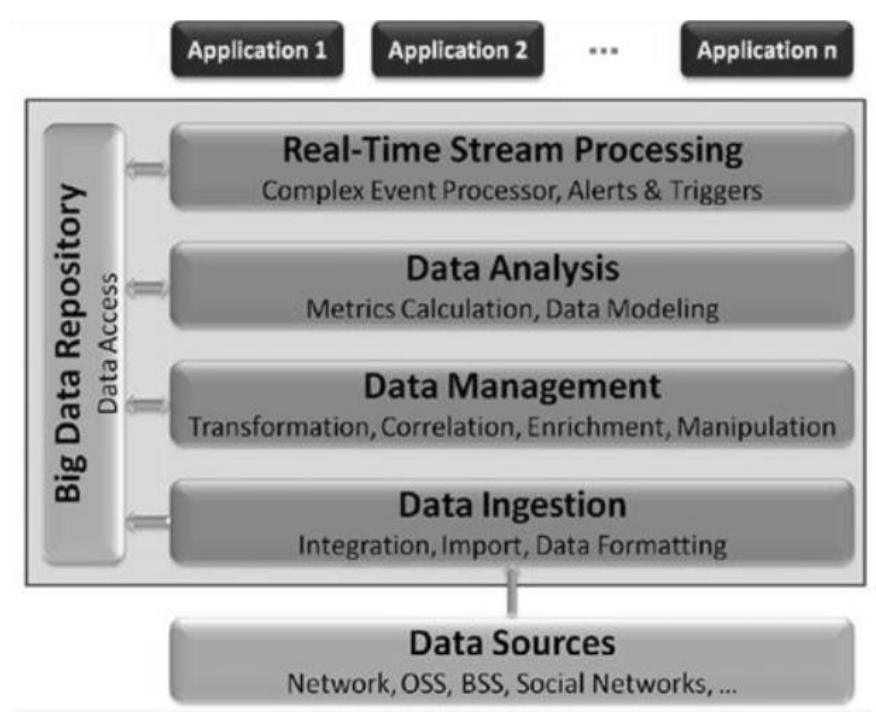

Figure 2. Big Data Analytics Architecture

\section{USING AI in SENSITIVE BUSINESS OPERATIONS}

The artificial intelligence rules define the way the online learning system assigned learning materials and exercises for the learner to follow [8]. These are the basic rules which we have carry out in our experiments, in which we find it effective in improving the learners understanding.

\subsection{Financial Industry}

Artificial intelligence (AI), along with other financial technology (fintech) innovations, are significantly changing the ways that financial business are being run, especially in the fields like trading and insurance, leading the traditional financial industry into a new era [9].

\section{Robots replacing humans}

Back in 2000, Goldman Sach's New York headquarters employed 600 traders, buying and selling stock on the orders of the investment bank's clients. Today there are just two equity traders left, as automated trading programs have taken over the rest of the work. Meanwhile, BlackRock, the world's biggest money manager, also cut more than 40 jobs earlier this year, replacing some of its human portfolio managers with artificially intelligent, computerized stock-trading algorithms. Those two big companies are not the only financial institutions replacing human jobs with robots. By 2025, AI technologies will reduce employees in the capital markets by 230,000 people worldwide, according to a report by the financial services consultancy Opimas [10]. 
Big new frontiers are only just beginning to opening up in fintech from AI, block chain and robotics to biometrics, augmented reality and cybersecurity. Among all the fintech innovations, the prospect of the block chain has the highest expectation. The block chain will change the way people store information, which is real, spreading fast and cross-border, and its 'de-centric' feature will allow everyone to know what other people are doing. The application of block chain in finance will once again bring about a revolutionary impact on the industry, just like AI does.

\subsection{Health Industry}

The Artificial intelligence (AI) is reshaping operations across industries. Arguably, healthcare is where these changes are poised to make the biggest impact - optimizing uptime and availability of the treatment solutions. Using AI-powered tools capable of processing large amounts of data and making real-time recommendations, healthcare organizations are learning they can reduce administrative waste in a number of areas, from medical equipment maintenance to hospital bed assignments [11].

Artificial intelligence is reinventing and reinvigorating modern healthcare through technologies that can predict, comprehend, learn and act. The ability of AI to transform clinical care has received widespread attention, but the technology's potential extends beyond patient care to processes across the spectrum of healthcare operations. In healthcare and other industries that depend on reliable equipment performance, few things are more disruptive than unexpected outages. These unplanned stops create costly emergency situations, such as extended downtime, rush delivery of parts and overtime to repair the equipment.

Facing pressure to improve profitability and efficiency, many healthcare organizations are turning to emerging technologies like AI and big data analytics to improve upon existing maintenance operations. Until recently, maintenance typically involved either reacting to an unexpected problem or adhering to a preventive maintenance schedule, which can sometimes result in unnecessary maintenance. line.

\subsection{Manufacturing Industry}

AI is core to manufacturing's real-time future. Real-time monitoring provides many benefits, including troubleshooting production bottlenecks, tracking scrap rates, meeting customer delivery dates, and more. It's an excellent source of contextually relevant data that can be used for training machine learning models. Supervised and unsupervised machine learning algorithms can interpret multiple production shifts' real-time data in seconds and discover previously unknown processes, products, and workflow patterns [12].

The manufacturing industry has exploited the use of AI technology, and in particular knowledgebased systems, throughout the manufacturing lifecycle. This has been motivated by the competitive challenge of improving quality while at the same time decreasing costs and reducing design and production time. Just-in-time manufacturing and simultaneous engineering have further required companies to focus on exploiting technology to improve manufacture planning and coordination, and on providing more intelligent processing in all aspects of manufacturing. The objective is to improve quality, to reduce costs, and to speed up the design and manufacturing process. 


\section{USing AI in CRISIS MANAgEMENT}

\subsection{Extreme Weather Forecast}

According to the UN Office for the Coordination of Human Affairs, in 2016 over 100 million people were affected by natural disasters including earthquakes, hurricanes and floods. Technology has a vital role to play in providing the appropriate situational awareness that then shapes practical, life-saving decisions for effective crisis management. These decisions may involve the evacuation of the most dangerous areas after an earthquake, or explore tactical options about how and where to position critical resources like medicine, food, clean water and shelter. Through utilising the data tweeted and texted by citizens in a crisis zone, rescuers have access to the knowledge needed to devise a strategy for immediate rescue attempts and for longer term help [13].

Issues can arise, however, due to the volume of available data, and high-quality filtering systems are needed to avoid using inaccurate data that could misdirect humanitarian aid, potentially wasting time, resources, and human trust in the system. Humanitarian responders may, understandably, question the specificity of information, therefore, building their trust and encouraging uptake of AI technology is a socially meaningful endeavour; without this, a system is unlikely to be adopted in the field. Machine learning, understood as the refinement of how AI 'learns' to use algorithms and other data, offers a solution to detecting key information taken from social media messages. Hence, researchers are focusing efforts on improving how the millions of messages are sifted by algorithms to overcome inaccuracy, ensuring that only the most important data is identified and shared.

\subsection{Man-Made Environmental Disaster}

The case of BP oil spill in 2010 provides an important example for understanding how these principles are valued by public opinion in a crisis situation, and how the communication actions by a corporation in this type of circumstances might have long-term effect on the brand image of the organization. On April 20, 2010, a BP's Deepwater Horizon oil rig exploded, causing what has been called the worst environmental disaster in U.S. history and taking the lives of $11 \mathrm{rig}$ workers. For 87 straight days, oil and methane gas spewed from an uncapped well-head, 1 mile below the surface of the ocean. The federal government estimated 4.2 million barrels of oil spilled into the Gulf of Mexico [14].

The accumulation of unsafe supervisory action had resulted in risk levels substantially increasing. Not only were risks increasing, but they were also incrementally becoming more aggressive in nature. For instance, one of the first acts of unsafe supervision is illustrated when BP neglected its responsibility of ensuring safety protocols were carried out after the completion of the Macondo Well. This was a major mistake on BP's part, violating safety protocols which may have identified the issues present with the cementing of the well. Should these issues have been identified sooner, the likeliness of the crisis happening would potentially be slim. In addition to this, there was also very little supervision during and after works were carried out. This can be attributed to the aforementioned organisational restructuring which created much confusion regarding who was accountable for the assurance of safety [15].

\subsection{Natural Disaster}

Researchers have found that AI can be used to predict natural disasters. With enormous amounts of good quality datasets, AI can predict the occurrence of numerous natural disasters, which can 
be the difference between life and death for thousands of people [16]. Some of the natural disasters that can be predicted by AI are:

\section{Earthquakes:}

AI systems can be trained with the help of seismic data to analyse the magnitude and patterns of earthquakes and predict the location of earthquakes and aftershocks.

\section{Floods:}

Various researchers and technology experts are developing AI-based applications with the help of rainfall records and flood simulations to predict and monitor flooding.

\section{Volcanic eruptions:}

AI-powered systems can accurately predict volcanic eruptions with the help of seismic data and geological information.

\section{Hurricanes:}

AI can use satellite to predict and monitor the path and intensity of hurricanes and tornadoes.

\section{CONCLuSions}

The study is assessing new frameworks for effective prevention measures and how AI can fit in and foster the early warning process. So further experiments and understanding the interrelation between AI and big data, what frameworks and systems that worked, and how AI can impact on different business operations whether by introducing new innovations that foster crisis management learning process and early prevention measures. The study from various reviews show promising results in using AI to learn specific industry big data and further evaluation and research is in progress.

\section{REFERENCES}

[1] M. K.Kakhani, S. Kakhani and S. R.Biradar, (2015). Research issues in big data analytics, International Journal of Application or Innovation in Engineering \& Management, 2(8), pp.228-232.

[2] A. Gandomi and M. Haider, (2015). Beyond the hype: Big data concepts, methods, and analytics, International Journal of Information Management, 35(2), pp.137-144.

[3] C. Lynch, (2008). Big data: How do your data grow?, Nature, 455, pp.28-29.

[4] X. Jin, B. W.Wah, X. Cheng and Y. Wang, (2015). Significance and challenges of big data research, Big Data Research, 2(2), pp.59-64.

[5] R. Kitchin, (2014). Big Data, new epistemologies and paradigm shifts, Big Data Society, 1(1).

[6] C. L. Philip, Q. Chen and C. Y. Zhang, (2014). Data-intensive applications, challenges, techniques and technologies: A survey on big data, Information Sciences, 275, pp.314-347.

[7] K. Kambatla, G. Kollias, V. Kumar and A. Gram, (2014). Trends in big data analytics, Journal of Parallel and Distributed Computing, 74(7), pp.2561-2573.

[8] S. Del. Rio, V. Lopez, J. M. Bentez and F. Herrera, (2014). On the use of mapreduce for imbalanced big data using random forest, Information Sciences, 285, pp.112-137.

[9] MH. Kuo, T. Sahama, A. W. Kushniruk, E. M. Borycki and D. K. Grunwell, (2014). Health big data analytics: current perspectives, challenges and potential solutions, International Journal of Big Data Intelligence, 1, pp.114-126.

[10] Xinhua China Daily, (18-Sep-2017). How is AI disrupting financial industry. http://www.chinadaily.com.cn/business/2017-09/18/content_32147126.htm 
International Journal of Computer Science, Engineering and Applications (IJCSEA)

Vol.11, No.5, October 2021

[11] Focus Elekta's Online Magazine, (2019). How AI is revolutionizing healthcare operations, https://focus.elekta.com/2019/10/how-ai-is-revolutionizing-healthcare-operations/

[12] Louis Columbus, (18-May-2020). 10 Ways AI is improving manufacturing in 2020, Forbes.https://www.forbes.com/sites/louiscolumbus/2020/05/18/10-ways-ai-is-improvingmanufacturing-in-2020/?sh=3530e5d1e85a

[13] Kejriwal M. \& Zhou P., (2019). SAVIZ: Interactive Exploration and Visualization of Situation Labeling Classifiers over Crisis Social Media Data, International Conference on Advances in Social Networks Analysis and Mining, Vancouver, Aug 27-30, pp705-708.

[14] National Commission, (2011). The Gulf Oil Disaster and the Future of Offshore Drilling.

[15] Dhaimaan Mahmud, (2019). Crisis Management Analysis of the BP Oil Spill, Birmingham Business School.

[16] Naveen Joshi, (15-Mar-2019). How AI can and will predict disasters, Forbes, https://www.forbes.com/sites/cognitiveworld/2019/03/15/how-ai-can-and-will-predictdisasters/?sh=7cddf40d5be2

\section{AUTHOR}

Prof. Yew Kee Wong (Eric) is a Professor of Artificial Intelligence (AI) \& Advanced Learning Technology at the HuangHuai University in Henan, China. He obtained his BSc (Hons) undergraduate degree in Computing Systems and a Ph.D. in AI from The Nottingham Trent University in Nottingham, U.K. He was the Senior Programme Director at The University of Hong Kong (HKU) from 2001 to 2016. Prior to joining the education sector, he has worked in international technology companies, Hewlett-Packard (HP) and Unisys as an AI consultant. His research interests include AI, online learning, big data analytics, machine learning, Internet of Things (IOT) and blockchain technology. 